\title{
Testing models for the formation of habitable planets
}

\author{
Yamila Miguel and Adrián Brunini \\ Facultad de Ciencias Astronómicas y Geofísicas (Universidad Nacional de La Plata) \\ Instituto de Astrofísica de La Plata (CCT La Plata, CONICET) \\ email: ymiguel@fcaglp.unlp.edu.ar
}

Abstract. We report on a statistical study of models for the formation of habitable planets.

Although planets with masses less than $15 \mathrm{M}_{\oplus}$ have already been detected, the sample is still not large enough and we also have to rely on what we know from our own Solar System, as well as on computational models, to gain a better understanding of the planetary formation process. Our principal aim is to analyse which factors will influence the formation of these habitable planets. To this end, we developed a simple code which allows us to form a large population of planets and study them statistically, analysing which parameters favor their formation.

We consider a population of 1000 different discs based on the Minimum Mass Solar Nebula, each one with a different star mass. The formation and evolution of several planets per disc are considered, taken into account the collisions among them as a source of potential growth. The embryos grow in the oligarchic growth regime and when a core reaches the critical mass for starting the gas accretion, the envelope starts to grow with a characteristic growth time proportional to $\left(\mathrm{M}_{p} / \mathrm{M}_{\oplus}\right)^{b}$. We perform simulations with two different values of $b$ : Model I assumes the value obtained by fitting the results of Fortier, Benvenuto \& Brunini (2007) $(b=-1.91)$, while Model II adopts the value used by Ida $\&$ Lin $(2004)(b=-3)$. We also consider type I and II regimes of embryos' migration, where we test different values for retarding the type I migration: $C_{m i g I}=1,10,100$.

As shown in Miguel \& Brunini $(2008,2009)$, the distributions of mass and semi-major axis strongly depend on the gas accretion model considered. We find that the population of habitable planets is larger when Model I is considered because the gas accretion rate is slower. As a consequence, runaway gas accretion is reached by the cores at a larger mass, so there are terrestrial planets that never reach this "crossover mass" and more planets form with small masses. We also find that when type I migration is slower, there are less planets reaching the inner edge of the disc, and we end up with a major population of terrestrial planets. We also analyse the effects produced by the collisions between the embryos. We find that there are a lot of planets beyond the stability limit, which are not gravitationally bound and should not be considered. As a result, the population of habitable planets is also strongly influenced by the collisions, a very important effect that must be taken into account when we analyse terrestrial planets.

\section{References}

Fortier, A., Benvenuto, O., \& Brunini, A. 2007, A\&A 473, 311

Ida, S. \& Lin, D. N. C. 2004, ApJ 604, 388

Miguel, Y. \& Brunini, A. 2008, MNRAS 387, 463

Miguel, Y. \& Brunini, A. 2009, MNRAS 392, 391 